\title{
Baciloscopia da conjuntiva no diagnóstico e acompanhamento de pacientes portadores de hanseníase
}

\author{
Conjunctival bacilloscopy in leprosy diagnosis and follow-up
}

\author{
Adriana Souza Moreira ${ }^{1}$ \\ Reg'ina Cândido Ribeiro dos Santos² \\ Ronaldo Rocha Bastos ${ }^{3}$ \\ José Vitor da Silva ${ }^{4}$ \\ Procópio Miǵuel dos Santos ${ }^{5}$
}

Trabalho realizado na Universidade de Brasília - UnB - Brasília (DF) - Brasil; Laboratório Central de Saúde Pública do Distrito Federal - Brasília (DF) - Brasil e Oculistas Associados de Brasília (DF) - Brasil.

Pós-Graduanda (Nível Doutorado) em Ciências da Saúde pela Universidade de Brasília - UnB - Brasília (DF) - Brasil.

Professora do Curso de Pós-Graduação da UnB - Brasília (DF) - Brasil

${ }^{3}$ PhD em Regional Science - Universidade de Liverpool, Inglaterra; Professor Adjunto IV - Departamento de Estatística - Instituto de Ciências Exatas - Universidade Federal de Juiz de Fora - UFJF - Juiz de Fora (MG) - Brasil. ${ }^{4}$ Técnico em Patologia Clínica do LACEN - Laboratório Central de Brasília (DF) - Brasil.

${ }^{5}$ Professor do Curso de Pós-Graduação da UnB - Brasília (DF) - Brasil.

Endereço para correspondência: Adriana Souza Moreira. Rua Francisco Vale, 73 - Juiz de Fora (MG) CEP 36016-270

E-mail: adriana.moreira@terra.com.br

Recebido para publicação em 08.06.2005

Última versão recebida em 08.05.2006

Aprovação em 18.05.2006

Dados parciais da tese a ser apresentada ao Curso de Pós-graduação em Ciências da Saúde, Universidade de Brasília (UnB), área de concentração Oftalmologia, para obtenção do Título de Doutor

Nota Editorial: Depois de concluída a análise do artigo sob sigilo editorial e com a anuência dos Drs. Eduardo Ferrari Marback e Sérgio Burnier sobre a divulgação de seus nomes como revisores, agradecemos sua participação neste processo.

\begin{tabular}{|c|}
\hline RESUMO \\
\hline $\begin{array}{l}\text { Objetivos: Estudar a presença do Mycobacterium leprae na conjuntiva } \\
\text { ocular e validar a baciloscopia de conjuntiva como teste diagnóstico e de } \\
\text { acompanhamento da hanseníase. Métodos: Foi realizado raspado de } \\
\text { conjuntiva tarsal superior em } 52 \text { pacientes portadores de hanseníase } \\
\text { recém-diagnosticados ( } 26 \text { multibacilares e } 26 \text { paucibacilares) no período } \\
\text { de julho a setembro de } 2004 \text { na clínica Oculistas Associados de Brasília e } \\
\text { foram analisados os resultados da baciloscopia de conjuntiva a fim de } \\
\text { compará-la com a baciloscopia da linfa (padrão-ouro). Resultados: A } \\
\text { avaliação da correlação entre o índice baciloscópico da conjuntiva (IBC) } \\
\text { e índice baciloscópico da linfa (IBL) medida pelo coeficiente de correlação } \\
\text { linear de Pearson é } 76,3 \% \text { (p<0,01). O teste qui-quadrado de Pearson } \\
\text { também evidenciou associação entre IBLe IBC (p<0,01). Oíndice } \kappa \text { (medida } \\
\text { de concordância kappa de Cohen) foi de } 0,615 \text { (p<0,01). Dos } 26 \text { casos com } \\
\text { IBL positivos, } 17 \text { tinham IBC positivo, demonstrando uma sensibilidade do } \\
\text { IBC de } 65,4 \% \text { (IC } 95 \% \text { - } 0,519-0,690 \text { ). Dos } 26 \text { casos de IBLnegativo, } 25 \text { eram } \\
\text { IBC negativo, revelando especificidade de } 96,2 \% \text { (IC } 95 \% \text { - } 0,826-0,998 \text { ). } \\
\text { O valor preditivo positivo é de } 94,4 \% \text { e o valor preditivo negativo é de } \\
73,5 \% \text { A acurácia do teste é de } 80,8 \% \text {. A razão de verossimilhança é } 17 \text {. } \\
\text { O tempo médio de negativação do bacilo no olho é de } 5 \text { meses (IC 95\%- } \\
3,57-6,43 \text { ). Conclusão: Sugerimos a utilização da combinação de baci- } \\
\text { loscopia da linfa e baciloscopia de conjuntiva para confirmação do diag- } \\
\text { nóstico e classificação da hanseníase e da baciloscopia de conjuntiva para } \\
\text { acompanhamento do tratamento poliquimioterápico na hanseníase. }\end{array}$ \\
\hline
\end{tabular}

Descritores: Hanseníase/diagnóstico; Conjuntiva/microbiologia

\section{INTRODUÇÃO}

A hanseníase é uma doença infecto-contagiosa, de evolução lenta, que se manifesta principalmente através de lesões na pele, nos nervos periféricos e nos olhos. É causada pelo Mycobacterium leprae, ou bacilo de Hansen, que é um parasita intracelular obrigatório que se instala no organismo da pessoa infectada, podendo se multiplicar. Dentre as pessoas que adoecem, algumas apresentam resistência ao bacilo, constituindo os casos paucibacilares (PB), que abrigam um pequeno número de bacilos no organismo; outras não apresentam resistência ao bacilo, que se multiplica no seu organismo passando a ser eliminado para o meio exterior, são os multibacilares (MB). O tratamento específico da pessoa com hanseníase, preconizado pelo Ministério da Saúde e pela Organização Mundial de Saúde é a poliquimioterapia (PQT), constituída pelo conjunto dos seguintes medicamentos: rifampicina, dapsona e clofazimina. A duração da PQT 
deve obedecer aos prazos estabelecidos: no mínimo 6 meses para os $\mathrm{PB}$ e 12 meses para os $\mathrm{MB}^{(1)}$.

O diagnóstico da hanseníase é feito a partir da apresentação clínica e/ou da identificação do M. leprae. A baciloscopia da linfa é o exame microscópico onde se observa o $M$. leprae diretamente nos esfregaços de raspados intradérmicos das lesões hansênicas ou de outros locais de coleta selecionados: lóbulos auriculares e/ou cotovelos ${ }^{(1-2)}$.

A baciloscopia da linfa é o exame complementar mais útil no diagnóstico, considerado padrão-ouro; é de fácil execução e baixo custo. Colhe-se o material a ser examinado (raspado de tecido dérmico) nos lóbulos das orelhas direita e esquerda, cotovelos direito e esquerdo e em lesão suspeita. A coloração é feita pelo método de Ziehl-Neelsen e apresenta-se o resultado sob a forma de índice baciloscópico da linfa (IBL), numa escala que vai de 0 a $6+$, dependendo do número de bacilos observados por campo à microscopia ${ }^{(3)}$. A baciloscopia da linfa positiva classifica o caso como $\mathrm{MB}$, independente do número de lesões ${ }^{(4)}$.

Os dois métodos de classificação, o baciloscópico e o clínico, utilizados na rotina operacional pelos profissionais de saúde responsáveis pelo diagnóstico e tratamento dos pacientes apresentam limitações e diferentes percentuais de sensibilidade e especificidade. Nesse sentido, há a necessidade de se pensar em novos critérios mais acurados para a classificação operacional em hanseníase. Estabelecer um referencial clínico simplificado de forma generalizada para a alocação dos pacientes nos esquemas PQT pode representar, em muitas situações, um retrocesso ${ }^{(5)}$.

O objetivo deste trabalho foi estudar a presença do Mycobacterium leprae na conjuntiva ocular e validar a baciloscopia de conjuntiva como teste diagnóstico e de acompanhamento da hanseníase.

\section{MÉTODOS}

No período de julho a setembro de 2004, 52 pacientes virgens de tratamento PQT foram atendidos na rede pública de saúde do DF (Hospital Regional da Asa Norte, Hospital Universitário da Universidade de Brasília e Centro de Saúde n.1) com diagnóstico de hanseníase, quando realizavam a coleta do material para baciloscopia da linfa e iniciavam imediatamente o tratamento com PQT. Neste mesmo momento era agendada consulta oftalmológica na clínica Oculistas Associados de Brasília onde foi realizada coleta do material para baciloscopia da conjuntiva. O intervalo entre o primeiro atendimento (diagnóstico, baciloscopia da linfa e início de tratamento PQT) e o exame oftalmológico com coleta da baciloscopia da conjuntiva não ultrapassou 7 dias em nenhum paciente (média de 5,21 dias).

Foi utilizada a seguinte técnica para raspado da conjuntiva tarsal superior: os olhos a serem examinados receberam 2 gotas de proparacaína (proximetacaina 0,5\%) como anestésico, em seguida a pálpebra superior foi evertida e a conjuntiva tarsal superior levemente raspada usando-se uma lâmina de bisturi descartável n.15 da região nasal para temporal. Em seguida foi feito um esfregaço do material na lâmina e a amostra foi deixada para secar no meio ambiente por 24 horas $^{(6)}$. Não houve sangramento ou corte na região raspada.

$\mathrm{O}$ raspado conjuntival, realizado primeiramente no olho direito $(\mathrm{OD})$ e, em seguida, no olho esquerdo (OE), foi realizado nas seguintes ocasiões: ao diagnóstico de hanseníase (quando os pacientes iniciaram a PQT) e de 2 em 2 meses até a alta do tratamento PQT ou negativação do bacilo, o que ocorrer por último.

Após 24 horas da realização do raspado conjuntival as lâminas foram encaminhadas ao laboratório central de saúde pública do DF onde foi corada pelo método de Ziehl-Neelsen e observada sob objetiva de imersão no microscópio por um único microscopista com notória experiência no estudo do $M$. leprae procedendo-se à contagem do índice baciloscópico da conjuntiva (IBC) segundo a escala de Ridley (de 0 a $6+)^{(7)}$. As lâminas foram identificadas com números, e não iniciais, de modo que apenas a médica oftalmologista responsável pelos esfregaços (ASM) pudesse correlacionar a lâmina à ficha de atendimento do paciente. O mesmo microscopista foi responsável pela baciloscopia da linfa desses pacientes, coletadas nos centros de saúde do qual os pacientes foram referenciados (Figuras 1 e 2).

Entre os pacientes estudados, $31(60 \%)$ eram do sexo masculino e $21(40 \%)$ do sexo feminino. Variavam entre 16 e 77 anos, com média de 39,4 anos.

Para avaliar a validade da baciloscopia da conjuntiva no diagnóstico e acompanhamento do tratamento dos portadores de hanseníase, os pacientes foram divididos, a partir da baciloscopia de linfa, em MB (26 casos) e PB (26 casos) e estabeleceu-se a comparação da baciloscopia da conjuntiva com o teste considerado padrão-ouro, a baciloscopia da linfa. A partir dessa comparação, foram calculadas a sensibilidade e a

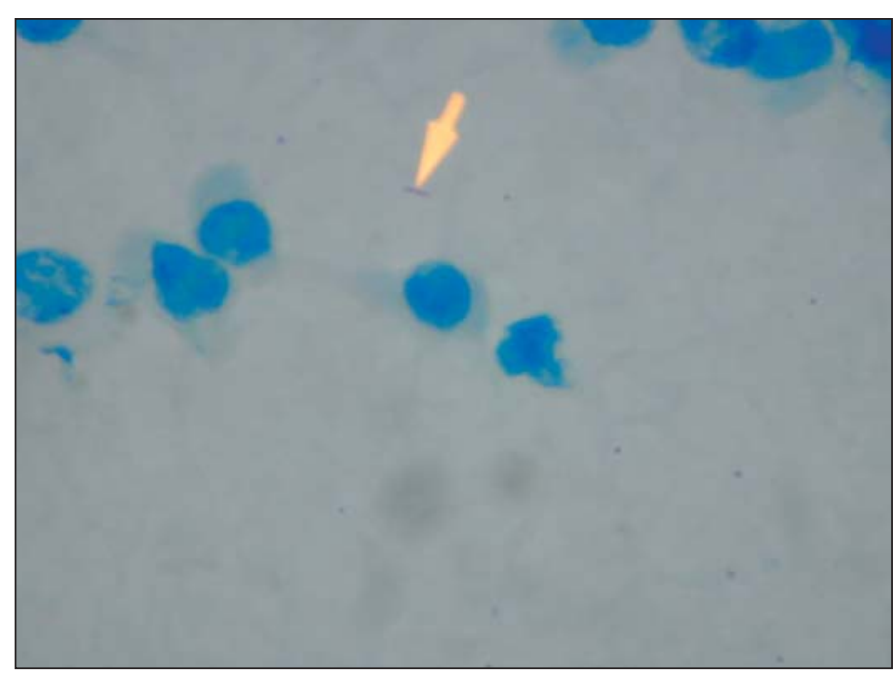

Figura 1 - Bacilo de Hansen (M. leprae) corado pelo método de ZiehlNeelsen no esfregaço de conjuntiva tarsal superior 


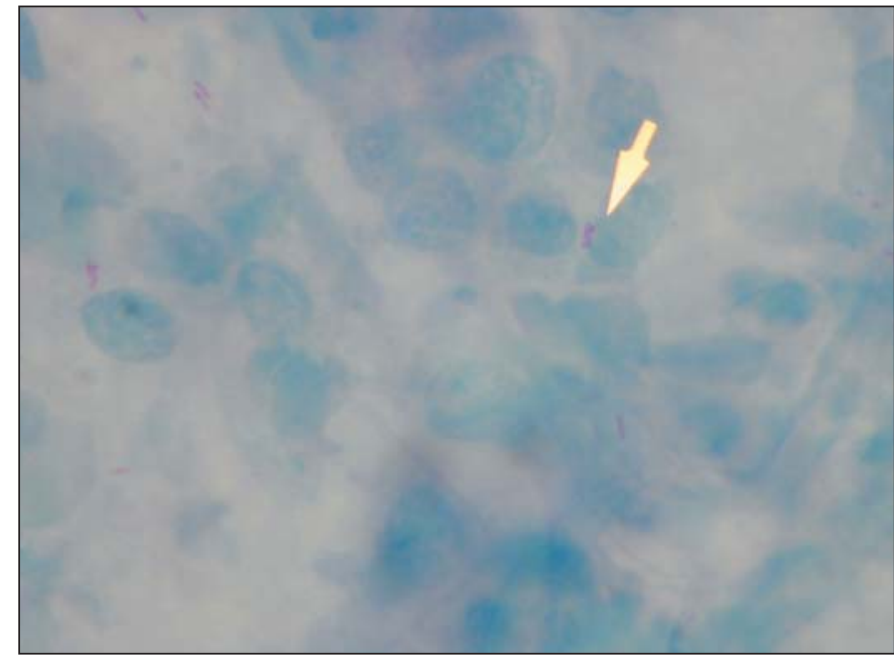

Figura 2 - Bacilo de Hansen (M. leprae) corado pelo método de ZiehlNeelsen no esfregaço de linfa

especificidade, bem como os valores preditivos positivo e negativo da baciloscopia da conjuntiva. Além disso, foi avaliada a concordância entre a baciloscopia da conjuntiva e a baciloscopia da linfa através do coeficiente de correlação linear de Pearson, teste qui-quadrado de Pearson e medida de

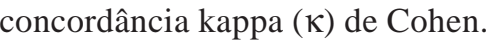

O protocolo desta pesquisa foi submetido e aprovado pelo Comitê de Ética da Universidade de Brasília sob o nํำ16/2004. Todos os pacientes compreenderam e assinaram Termo de Consentimento Livre e Esclarecido para participar do estudo.

\section{RESULTADOS}

A avaliação da correlação entre o IBC e IBL medida pelo coeficiente de correlação linear de Pearson foi 76,3\% ( $\mathrm{p}<0,01)$. $\mathrm{O}$ teste qui-quadrado de Pearson também evidenciou associação entre IBL e IBC $(\mathrm{p}<0,01)$. $O$ índice $\kappa$ (medida de concordância kappa de Cohen) foi de 0,615 ( $p<0,01$ ), correspondendo a uma boa concordância segundo a escala de concordância do kappa. Dos 26 casos com IBL positivos, 17 tinham IBC positivo, demonstrando uma sensibilidade do IBC de $65,4 \%$ (IC 95\% - 0,519-0,690). Dos 26 casos de IBL negativo, 25 eram IBC negativo, revelando especificidade de 96,2\% (IC 95\% - 0,826-0,998). O valor preditivo positivo foi de 94,4\% e o valor preditivo negativo foi de $73,5 \%$. A acurácia do teste é de $80,8 \%$. A razão de verossimilhança é 17 . O tempo médio de negativação do bacilo no olho, entre os pacientes com IBC inicial positivo, foi de 5 meses (IC 95\% - 3,57-6,43). Um caso $\mathrm{MB}$ não negativou até a data da alta da PQT, preconizada em 12 meses pelo Ministério da Saúde. Entre os 18 pacientes com IBC positivo apenas $6(33,3 \%)$ apresentavam manifestação oftalmológica relacionada à hanseníase (2 casos de catarata pós-uveíte anterior sendo que 1 deles também tinha ectrópio, 2 casos de ceratoconjuntivite sicca, 1 caso de madarose total de supercílios e 1 caso de hipoestesia corneana).

\section{DISCUSSÃO}

O quadro clínico oftalmológico na hanseníase é causado por ação direta do bacilo no olho além de lesões do nervo facial, lesões do nervo trigêmeo (ramo oftálmico) e lesões por hipersensibilidade ${ }^{(8)}$. As manifestações oculares mais freqüentes em casos de hanseníase são: hipoestesia corneana, madarose, lagoftalmo, ectrópio, catarata, iridociclite e opacidades corneanas $^{(8-12)}$

Lesões oculares na hanseníase se desenvolvem gradualmente e insidiosamente sem sintomas ou sinais evidentes. Pouco material é disponível nas lesões precoces/iniciais para estudo histopatológico devido ao risco envolvido nos procedimentos de biópsia do olho sendo a patologia e patogênese das lesões oculares inadequadamente estudadas e pobremente entendidas. O pouco material disponível é proveniente de olhos obtidos em autópsias ou de pacientes cujos olhos foram enucleados após complicações avançadas e irreversíveis causando dor intratável e/ou cegueira ${ }^{(6)}$.

Chaves et al. afirmam que os bacilos invadem o bulbo ocular e multiplicam-se no corpo ciliar, espalhando-se a partir daí para as estruturas intra-oculares, sobretudo as do segmento anterior, podendo também comprometer o segmento posterior ${ }^{(13)}$.

Apenas 6 dos 18 pacientes com IBC positivo apresentavam manifestação oftalmológica relacionada à hanseníase, portanto nos 12 casos restantes o bacilo de Hansen estava presente na conjuntiva ocular sem causar qualquer doença ocular. Vale, portanto, resaltar a importância do acompanhamento periódico destes pacientes já que o bacilo está presente na conjuntiva ocular, podendo levar a manifestações oculares a qualquer momento. Acredita-se que os pacientes com IBC positivo poderiam se beneficiar com tratamento tópico (colírio de rifampicina, por exemplo) no auxílio à eliminação do bacilo no olho. Um estudo detalhado sobre a questão poderia elucidar melhor tal afirmação.

$\mathrm{Na}$ presente pesquisa foi observada forte correlação entre o IBC e IBL, evidenciados pelo coeficiente de correlação linear de Pearson, teste qui-quadrado de Pearson e medida de concordância kappa de Cohen. Conclui-se que o IBC reflete qualitativamente e quantitativamente a presença do $M$. leprae na linfa. Além disso, a Razão de verossimilhança revelou-nos que um IBC positivo é 17 vezes mais provável de ocorrer em pacientes com IBL positivo que em pacientes IBL negativos.

Campos et al. examinaram 56 pacientes MB em Passos, Minas Gerais, através da técnica do raspado de conjuntiva tarsal superior. Alguns pacientes nunca haviam sido tratados para hanseníase enquanto outros estavam recebendo PQT. O raspado da conjuntiva tarsal superior mostrou bacilos de Hansen em 21 casos. Os bacilos estavam no epitélio conjuntival. Bacilos morfologicamente intactos foram encontrados apenas em casos não tratados. Apesar de não encontrarem relação direta entre os 2 índices, os autores afirmaram que o IBC tende a ser maior quando IBL é alto e valores positivos de IBC foram associados com valores altos de IBL, em concordância com presente estu- 
do. Os autores acreditam que quando os valores do IBL são maiores que 3+ os valores do IBC também aumentam ${ }^{(6)}$.

Pode-se demonstrar que a baciloscopia de conjuntiva apresenta uma sensibilidade de $65,4 \%$, especificidade de $96,2 \%$, valor preditivo positivo de $94,4 \%$ e o valor preditivo negativo de 73,5\% (Tabela 2). Não foi possível comparar este resultado com outras pesquisas porque não foi encontrado, na literatura pesquisada, qualquer trabalho que avaliasse a baciloscopia de conjuntiva como teste diagnóstico exclusivamente em pacientes virgens de tratamento específico para hanseníase.

A necessidade do exame baciloscópico de linfa para a classificação dos casos de hanseníase foi avaliada em um estudo na Índia, onde os resultados demonstraram especificidade de 98,1\% com a classificação baciloscópica e de 41,3\% quando baseada em critérios clínicos ${ }^{(14)}$. No Brasil, Andrade et al. realizaram um trabalho comparando diferentes classificações utilizadas pelas coordenações dos programas de controle em diferentes unidades da federação. Segundo esses autores, se a baciloscopia fosse o único critério para alocação dos pacientes, $20 \%$ de casos MB seriam classificados como $\mathrm{PB}^{(15)}$. Em estudo similar, foi analisada uma coorte de 2.664 casos; comparando-se a classificação pelo número de lesões cutâneas com os resultados dos exames baciloscópicos, foi demonstrado que o método clínico apresentava sensibilidade de $89 \%{ }^{(16)}$. Resultados semelhantes foram obtidos por Bührer-Sékula et al. quando analisaram 264 pacientes, alcançando sensibilidade de $85 \%$ e especificidade de $80 \%$ do método clínico, bem como o valor preditivo positivo de $81 \%$ e o valor preditivo negativo de $85 \%$. Nesse mesmo estudo o índice $\kappa$ foi de 0,66 , correspondendo a um bom nível de concordância com a taxa de $83,03 \%$ entre as classificações clínica e baciloscópica ${ }^{(17)}$.

A dificuldade de operacionalização de quaisquer métodos complementares à avaliação clínica torna esse objetivo difícil

\begin{tabular}{|lcccc|}
\hline \multirow{4}{*}{ Tabela 1. Valores cruzados de índice baciloscópico da conjuntiva } \\
& & \multicolumn{3}{c}{ IBL e índice baciloscópico da linfa (IBL) } \\
\cline { 3 - 5 } & & Positivo & Negativo & Total \\
IBC & Positivo & 17 & 1 & 18 \\
& Negativo & 9 & 25 & 34 \\
Total & & 26 & 26 & 52 \\
\hline
\end{tabular}

Tabela 2. Validade do índice baciloscópico da conjuntiva como teste diagnóstico em comparação ao índice baciloscópico da linfa

\begin{tabular}{|lcc|} 
& (padrão-ouro) \\
Odds ratio & Valor & $\begin{array}{c}\text { Intervalo de } \\
\text { confiança de 95\% }\end{array}$ \\
Risco relativo & 47,222 & $5,123-1117,540$ \\
Kappa & 3,568 & $2,035-4,211$ \\
Sensibilidade & 0,615 & $0,345-0,688$ \\
Especificidade & 0,654 & $0,519-0,690$ \\
Valor preditivo positivo & 0,962 & $0,826-0,998$ \\
Valor preditivo negativo & 0,944 & $0,749-0,997$ \\
\hline
\end{tabular}

de ser alcançado, o que justifica ainda mais a busca de métodos acurados e mais simplificados ${ }^{(5)}$.

A baciloscopia da conjuntiva é apresentada neste estudo como um teste simples, indolor e de baixo custo, capaz de auxiliar o diagnóstico inicial da hanseníase e até mesmo o acompanhamento da eliminação do $M$. leprae durante e após a PQT. Além disso, a baciloscopia da conjuntiva vem auxiliar na compreensão dos mecanismos de invasão do bacilo no olho e da etiopatogenia das diversas manifestações oculares da hanseníase.

De acordo com os resultados do presente trabalho, a baciloscopia de conjuntiva demonstrou ser um teste de alta especificidade e alto valor preditivo positivo quando comparado a outros critérios diagnósticos citados na literatura, sendo um teste de grande valia no auxílio do diagnóstico da hanseníase.

A especificidade é a capacidade que o teste tem de detectar os verdadeiros negativos, isto é, de diagnosticar corretamente os indivíduos PB. Especialmente na hanseníase tal característica reveste-se de importância pois a recomendação de tratamento PQT para pacientes PB é de 6 doses em contraste com as 12 doses que devem ser administradas aos MB. Sabese que as drogas que compõem a PQT tem efeitos colaterais relevantes, não devendo ser administradas por um período de tempo superior ao necessário. Portanto, um teste com grande especificidade para hanseníase impede que grande número de pacientes $\mathrm{PB}$ seja incluído em esquema de tratamento para $\mathrm{MB}$, tendo conseqüências sérias do ponto de vista individual e de saúde pública: haveria exposição desnecessária dos pacientes a drogas potencialmente tóxicas; o diagnóstico motivaria problemas de ordem psicossocial em face do estigma; e o programa de controle teria seus custos aumentados pelo desperdício das drogas erroneamente utilizadas ${ }^{(18)}$.

O valor preditivo positivo é a proporção de doentes entre os considerados positivos ao teste ${ }^{(19)}$. Neste caso, a proporção de MB entre os pacientes com IBC positivo, resultando em poucos falso-positivos. Conclui-se que um resultado IBC positivo indica administração de PQT em 12 doses e classificação do paciente como MB. Entre os 52 pacientes desta pesquisa, 42 tiveram seu diagnóstico correto confirmado pela BC, revelando acurácia de $80,8 \%$ neste teste.

O tempo médio de negativação do bacilo no olho foi de 5 meses. Um caso não negativou até a data da alta da PQT. Principalmente do ponto de vista oftalmológico, tal dado é de grande relevância. Afirma-se que muitas das manifestações oftalmológicas da hanseníase são causadas por invasão direta do bacilo no olho ${ }^{(6)}$. Se for considerado que a negativação do bacilo leva em média 5 meses para ocorrer faz-se necessário alertar os profissionais envolvidos no acompanhamento de portadores de hanseníase para a possibilidade da ocorrência de tais manifestações mesmo após iniciado o tratamento. Deve-se alertar para a possibilidade de não negativação do bacilo no olho mesmo após a alta medicamentosa.

Sugere-se, portanto, a realização de baciloscopia de conjuntiva nos pacientes no momento da alta medicamentosa com o intuito de evitar a possibilidade de desenvolvimento tardio de incapacidades visuais nestes pacientes. Enfatiza-se ainda 
que a baciloscopia de conjuntiva pode ser utilizada no momento da alta do tratamento também com o intuito de sinalizar a possibilidade de não negativação do bacilo em outras regiões do organismo já que expusemos anteriormente sua forte correlação com a baciloscopia da linfa. Sugere-se também que futuras pesquisas avaliem a possibilidade de tratamento tópico (colírio de rifampicina, por exemplo) com o objetivo de eliminação mais precoce do M. leprae na conjuntiva ocular.

Uma sugestão que deve ser objeto de reflexão é a utilização da combinação de baciloscopia da linfa e baciloscopia de conjuntiva para confirmação do diagnóstico e classificação da hanseníase e da baciloscopia de conjuntiva para acompanhamento do tratamento PQT.

\section{ABSTRACT}

Purpose: To identify Mycobacterium leprae in ocular conjunctivae and evaluate conjunctival bacilloscopy as leprosy diagnosis and follow-up test. Methods: We used the superior tarsal conjunctiva scraping technique in 52 newly diagnosed leprosy patients between July and September 2004 at the "Oculistas Associados de Brasília", in Brasília, DF, Brazil, and compared the conjunctival bacilloscopy with skin bacilloscopy. Results: Both the conjunctival bacilloscopy index (CBI) and skin bacilloscopy index (SBI) showed correlation since Pearson's $r$ and Cohen kappa measure of agreement were respectively $76.3 \%$ $(\mathrm{p}<0.01)$ and $0.615(\mathrm{p}<0.01)$. Pearson chi-square also evidenced the correlation $(\mathrm{p}<0.01)$. Comparison of CBI and SBI ( 17 cases of positive CBI out of $26 \mathrm{SBI}$ ) gives a sensitivity of $65.4 \%$ (CI95\% - 0.519-0.690); 25 cases of positive CBI out of 26 cases of negative SBI give a specificity of $96.2 \%$ (CI95\% - 0.826-0.998); positive predictive value of $94.4 \%$; negative predictive value of $73.5 \%$, and accuracy of $80.8 \%$. Likelihood ratio was 17 . Mean time to eliminate the bacilli from conjunctiva was 5 months (CI95\% - 3.57-6.43). Conclusion: We suggest the use of conjunctival bacilloscopy in addition with skin bacilloscopy to confirm leprosy diagnosis. Conjunctival bacilloscopy may also be useful for treatment follow-up in leprosy.

Keywords: Leprosy/diagnosis; Conjunctiva/microbiology

\section{REFERÊNCIAS}

1. Brasil. Ministério da Saúde. Secretaria de Políticas de Saúde. Guia para o controle da hanseníase. Brasília: Ministério da Saúde; 2002.

2. Stretch R. Presentation and treatment of Hansen's disease. Nurs Times. 1999; 95(29):46-7.

3. Araujo MG. Hanseníase no Brasil. Rev Soc Bras Med Trop. 2003;36(3):373-82.

4. Brasil. Ministério da Saúde. Secretaria de Políticas de Saúde. Departamento de Atenção Básica. Área Técnica de Dermatologia Sanitária. Legislação sobre o controle da hanseníase no Brasil. Brasília: Ministério da Saúde; 2002.

5. Crippa ILF, Schettini AP, Pennini SN, Schettini MC, Rebello PFB. Correlação clínico-laboratorial baseada em dados secundários dos casos de hanseníase atendidos no período de 01/2000 a 03/2001 na Fundação Alfredo da Matta, Manaus-AM, Brasil. An Bras Dermatol. 2004;79(5):547-54.

6. Campos WR, Rodrigues CA, Orefice F, Monteiro LG. Identification of M. leprae in conjunctiva of leprosy patients using the superior tarsal conjunctiva scrape technique. Indian J Lepr. 1998;70(4):397-403.

7. WHO. Microbiology of Mycobacterium leprae [text on the Internet]. World Health Organization, 2004. [cited 2005 Jun 26]. Available form: <http:// www.who.int/lep/disease/Microbiology/frmain.htm>

8. Monteiro LG, Campos WR, Oréfice F, Grossi MAF. Estudo das alterações oculares em hansenianos de controle ambulatorial. Rev Bras Oftalmol. 1992; 51(3): 167-70.

9. Srinivasan H. Ocular morbidity in leprosy-affected persons. Indian J Lepr. 1998;70(1):1-4.

10. Oliveira Neto HL, Silva JLM, Manso PG, Botene IM, Sartori MBF. Envolvimento ocular na hanseníase: estudo em pacientes de ambulatório. Arq Bras Oftalmol. 1996;59(2):162,164,166 passim.

11. Maradei J, Santos PM, Santos RCR, Olivalves SMR, Abreu MT. Complicações oculares como causa de incapacidade visual na hanseníase. Arq Bras Oftalmol. 1998;61(1):11-4.

12. Job CK, Ebenezer GJ, Thompson K, Daniel E. Pathology of eye in leprosy. Indian J Lepr. 1998;70(1):79-91.

13. Chaves C, Cohen J, Ribeiro E. Manifestações oculares em doenças tropicais I: lepra. Rev Bras Oftalmol. 1992;51(1):21-4.

14. Groenen G, Saha NG, Rashid MA, Hamid MA, Pattyn SR. Classification of leprosy cases under field conditions in Bangladesh. I. Usefulness of skinsmear examinations. Lepr Rev. 1995;66(2):126-33.

15. Andrade VLG, Moreira TA, Avelleira JCR, Marques AB, Bayona M. Paucibacilar ou multibacilar? Uma contribuição para os serviços de saúde. Hansen Int. 1996;21(2):6-13.

16. Croft RP, Smith WC, Nicholls P, Richardus JH. Sensitivity and specificity of methods of classification of leprosy without use of skin-smear examination. Int J Lepr Other Mycobact Dis. 1998;66(4):445-50.

17. Bührer-Sékula S, Sarno EN, Oskam L, Koop S, Wichers I, Nery JA, et al. Use of ML dipstick as a tool to classify leprosy patients. Int J Lepr Other Mycobact Dis. 2000;68(4):456-63.

18. Van Brakel WH, de Soldenhoff R, McDougall AC. The allocation of leprosy patients into paucibacillary and multibacillary groups for multidrug therapy, taking into account the number of body areas affected by skin, or skin and nerve lesions. Lepr Rev. 1992;63(3):231-46.

19. Pereira MG. Epidemiologia: teoria e prática. Rio de Janeiro: Guanabara Koogan; 1995. 